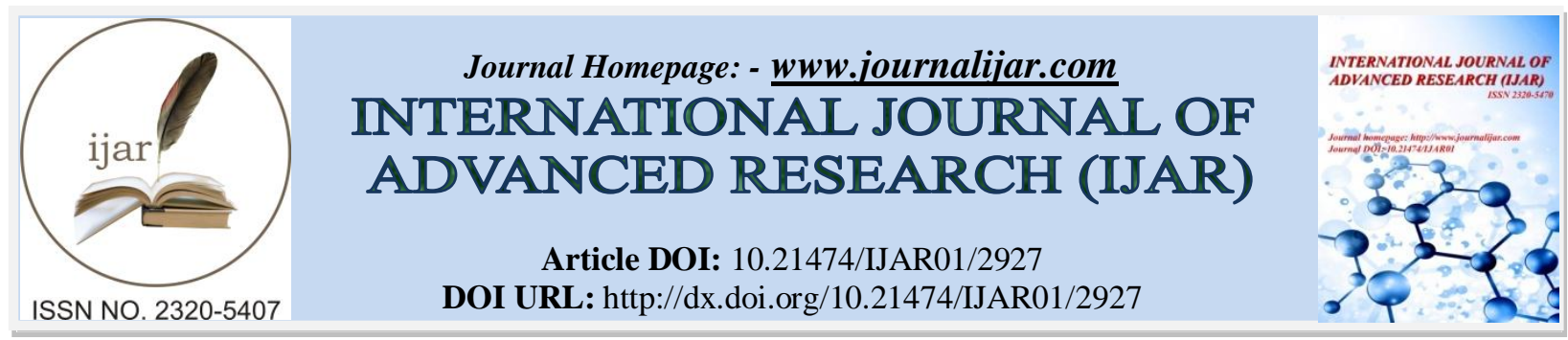

RESEARCH ARTICLE

\title{
EFFECT OF MCKENZIE EXTENSION BIAS EXERCISES WITH THAT OF STRENGTHENING EXERCISES IN LOW BACK PAIN IN POWER LIFTERS.
}

Gaurai Gharote ${ }^{1}$, Ayena Das ${ }^{2}$, Ujwal Yeole ${ }^{3}$, Rasika Panse ${ }^{4}$, Shweta Kulkarni ${ }^{4}$ and Pournima Pawar ${ }^{4}$.

1. Assistant professor, Tilak Maharashtra Vidyapeeth, Pune.

2. (Intern) Tilak Maharashtra Vidyapeeth, Pune.

3. Principal, Tilak Maharashtra Vidyapeeth, Pune.

4. Assistant professor, Tilak Maharashtra Vidyapeeth, Pune.

\section{Manuscript Info}

(..........................

Manuscript History

Received: 24 November 2016

Final Accepted: 25 December 2016

Published: January 2017

Key words:-

McKenzie exercises, Back

strengthening, Low back pain, Power

lifters.

\section{Abstract}

Background: Chronic nonspecific low back pain (i.e., low back pain of at least 12 weeks' duration and without a specific cause) is one of the most common health conditions worldwide. Chronic low back pain is highly associated with disability, emotional changes, and work absenteeism.Power lifting has many potential benefits for spinal health, but also has the potential to create or worsen back injuries. Extending or flexing the back muscles against resistance during power lifting may result in a number of injuries, including muscle strain and ligament injury. Currently no randomized studies examining the effect of this method for patient with low back pain have been published so the need of study is to find out the effect of McKenzie extension bias treatment method with that of back strengthening exercises in low back pain.

Method: A randomized controlled comparative trial with a 4 weeks treatment period was conducted. 40 consecutive power lifters both male and female between the age group 20-35 years with low back pain and at least 8 weeks duration of symptoms were randomized into two groups: Group A was treated with the McKenzie method and Group B was treated with back strengthening exercises. Oswestry scale and VAS scale was taken pre-treatment and post treatment. Results were recorded at the end of the treatment.

Result: The correlation coefficient $r=0.619$ is positive therefore there is improvement by using the Back strengthening treatment method in power lifters. The correlation coefficient $\mathrm{r}=0.821$ is positive and therefore shows more improvement by using McKenzie treatment method. Coefficient of variation of McKenzie treatment method that is 11.9335 is better than coefficient of variation of back strengthening method that is 14.0311 since coefficient of variation of McKenzie is smaller than back strengthening.

Conclusion- It was found that both McKenzie treatment method and back strengthening exercises were effective for Low back pain but McKenzie treatment method is more effective than back strengthening method.

Copy Right, IJAR, 2016,. All rights reserved.

Corresponding Author:- Gaurai Gharote.

Address:- Assistant professor, Tilak Maharashtra Vidyapeeth, Pune. 


\section{Introduction:-}

Chronic nonspecific low back pain (i.e., low back pain of at least 12 weeks' duration and without a specific cause) is one of the most common health conditions worldwide. Chronic low back pain is highly associated with disability, emotional changes, and work absenteeism. (1)

A typical response to experiencing back pain is to take it easy - either staying in bed or at least stopping any activity that is at all strenuous. While this approach is understandable and may even be recommended in the short term, when done for more than a day or two it can actually undermine healing. Instead, active forms of back exercises are almost always necessary to rehabilitate the spine and help alleviate back pain. When done in a controlled, gradual, and progressive manner, active back exercises distribute nutrients into the disc space and soft tissues in the back to keep the discs, muscles, ligaments and joints healthy. Consequently, a regular routine of lower back exercises helps patients avoid stiffness and weakness, minimize recurrences of lower back pain, and reduce the severity and duration of possible future episodes of low back pain. Depending upon the patient's specific diagnosis and level of pain, the back pain exercises and rehabilitation programs will be very different, so it is important for patients to see a spine specialist trained to develop an individualized program of back exercises and to provide instruction on using the correct form and technique. To be effective, a patient's program of back exercises should be comprehensive, working the whole body even if it targets the back. Two back exercises commonly advised by physical therapists to treat back pain are McKenzie exercises and dynamic lumbar stabilization.

Power lifting is a strength sport that consists of three attempts at maximal weight on three lifts: squat, bench press, and deadlift. It evolved from a sport known as "odd lifts", which followed the same three-attempt format but used a wider variety of events. Like most forms of exercise, power lifting has many potential benefits for spinal health, but also has the potential to create or worsen back injuries. Extending or flexing the back muscles against resistance (the weight) during power lifting may result in a number of injuries, including muscle strain and ligament injury. (2)

Disability related to lumbo-sacral pain is a major problem across many regions. Two recent consensus reports on the treatment of patients with chronic low back pain recommended back training programs as effective treatment for reduction of disability and improvement of physical function. (3)

Many back exercises can help strengthen the spinal column and the supporting muscles, ligaments and tendons. Most of these back exercises focus not only on the back, but also the abdominal (stomach) muscles and gluteus (buttocks) and hip muscles. Taken together, these strong 'core' muscles can provide back pain relief because they provide strong support for the spine, keeping it in alignment and facilitating movements that extend or twist the spine. Two of the most well-known back strengthening exercises are: McKenzie exercises and Dynamic Lumbar Stabilization. These back exercises are generally first learned by working with a physical therapist who can demonstrate the exercises and correct a patient's form to ensure strengthening and/or back pain relief is achieved. Although McKenzie exercises and dynamic lumbar stabilization exercises tend to be used for specific conditions, the two forms of physical therapy exercise may also be combined when appropriate.

Exercise therapy by the McKenzie method is a popular treatment for low back pain among physical therapists.It is grounded in finding a causeand effectrelationship between the positions the patient usually assumes while sitting, standing or moving, and the location of pain as a result of those positions or activities. The therapeutic approach requires a patient to move through a series of activities and test movements to gauge the patient's pain response. There are three types of classifications:

- Postural Syndrome: is the result of prolonged positions or postures that can affect muscles, tendons, or joint surfaces. Pain may be local and reproducible when end range positions, such as slouching, are maintained for sustained periods of time.

- Dysfunction Syndrome: Implies some sort of adaptive shortening, scarring or adherence of connective tissue causing discomfort. A dysfunction may be intermittent or chronic, but its hallmark is a consistent movement loss and pain at the end range of movement. When the patient moves away from end range their pain is decreased.

- Derangement Syndrome: is the most common syndrome of the three. Certain movements and particular movement patterns can cause more sensitivity. Such as a flexion or extension, the symptoms (low back pain) become either more central (just in the low back) or less intense. 
In this study, patients treated were suffered from derangement syndrome due to lifting heavy weight and postural deformity.

The McKenzie assessment consists of taking a patient history and performing a physical exam. Both are used to gauge the degree of impairment as well as identify any red flags that might be contrary to exercise-based treatment (e.g. fracture, tumor, infections, or systemic inflammatory disease). During the McKenzie physical examination, patients are taken through movements that help classify the patient and determine the best treatment approach. The movements are intended to either increase or decrease symptoms. For example, patients may be asked to perform single and/or repeated flexion or extension movements forward and backward.

McKenzie treatment prescribes a series of individualized exercises. The emphasis is on active patient involvement, which minimizes the number of visits to the clinic. It is important for the patient undergoing the McKenzie method of treatment to know what to do to manage any future low back pain episodes. The McKenzie approach then uses that information to develop an exercise program designed to centralize or alleviate the pain. The goal of McKenzie Method is to centralize the pain or move the pain from the leg into the low back. Although no randomized controlled trials have been published to support the effectiveness of the method. (4)

Currently no randomized studies examining the effect of this method for patient with low back pain have been published so the need of study is to find out the effect of McKenzie extension bias treatment method with that of back strengthening exercises in low back pain.

\section{Materials and Methodology:-}

Materials: Pen, paper, Questionnaire, outcome measures: Oswestry disability index and visual analog scale

\section{Methodology:-}

The study was performed across the gymnasiums of pune where the power lifters who had left the gymnasium were called for the assessment and treatment purpose. . Subjects were taken between the age group of 20- 35 years, both male and female. Total 89 Power lifters were called to the gym for assessment from which 27 power lifters were excluded due to recent history of fracture and previous history of Spondylolisthesis. 12 power lifters did not showed up later. Finally 40 power lifters agreed to take the treatment and filled the consent form. Those 40 power lifters were divided into 2 groups: 20 were treated by using McKenzie method and remaining 20 were treated by back strengthening exercises. Visual Analog scale and Oswestry Scale was taken before and after the treatment. The treatment period was 8 weeks and after that home exercise protocol was given. It was seen that there was improvement in the patient's low back pain as the pain score reduced in VAS scale. Similarly in Oswestry scale the percentage of pain reduced when compared to the pain patient felt before treatment. Complete assessment was done. Subjects from this study were selected from a subject group normally considered suitable for strengthening training in as much as all patients with clinical signs of affected nerve root were excluded.

\section{Procedure:-}

For study research, 40 subjects were selected. According to the inclusion and exclusion, each patient was explained the purpose of the study and a consent form was given to be filled by the patient. Two treatment protocols were followed: McKenzie Exercise protocol and Backstrengthening exercises. Oswestry disability scale and Visual analog scale was taken before and after the treatment session. 


\section{Results:-}

Result 1:- Improvement in back pain by using VAS in McKenzie treatment method.

Graph:-

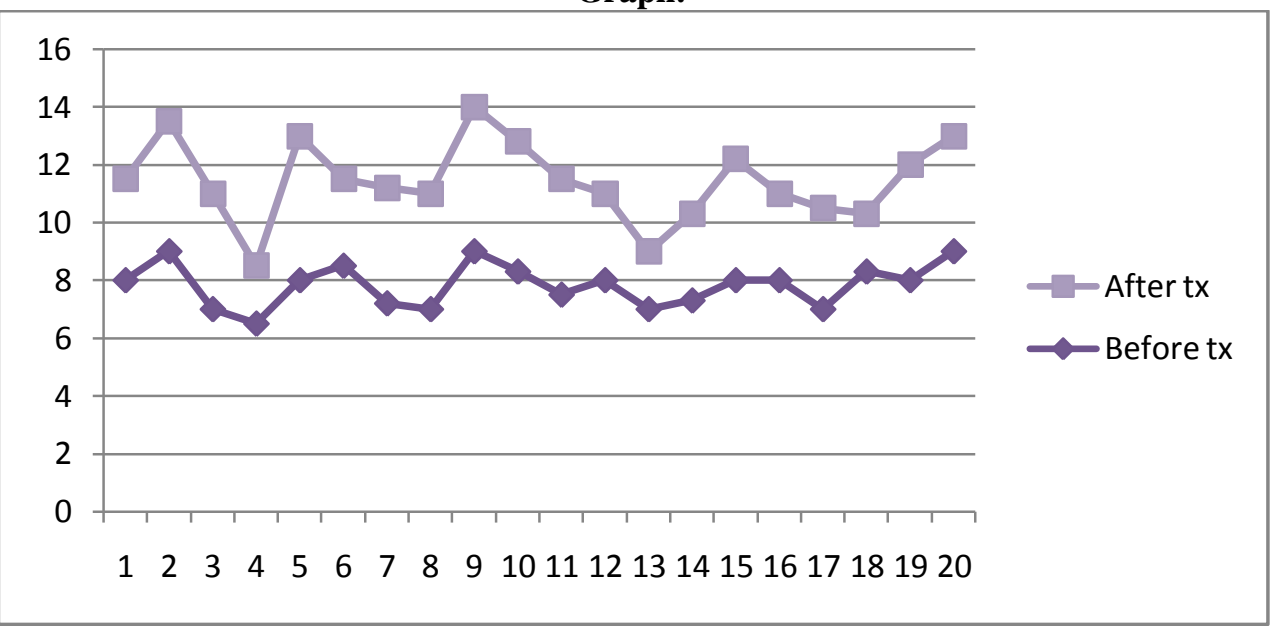

Interpretation: Correlation coefficient $r=0.821$ is more than 0.5 , therefore it is positive so there is improvement seen in VAS by using McKenzie treatment method.

Result 2:- Improvement in back pain by using VAS in Back strengthening treatment method.

\section{Graph:-}

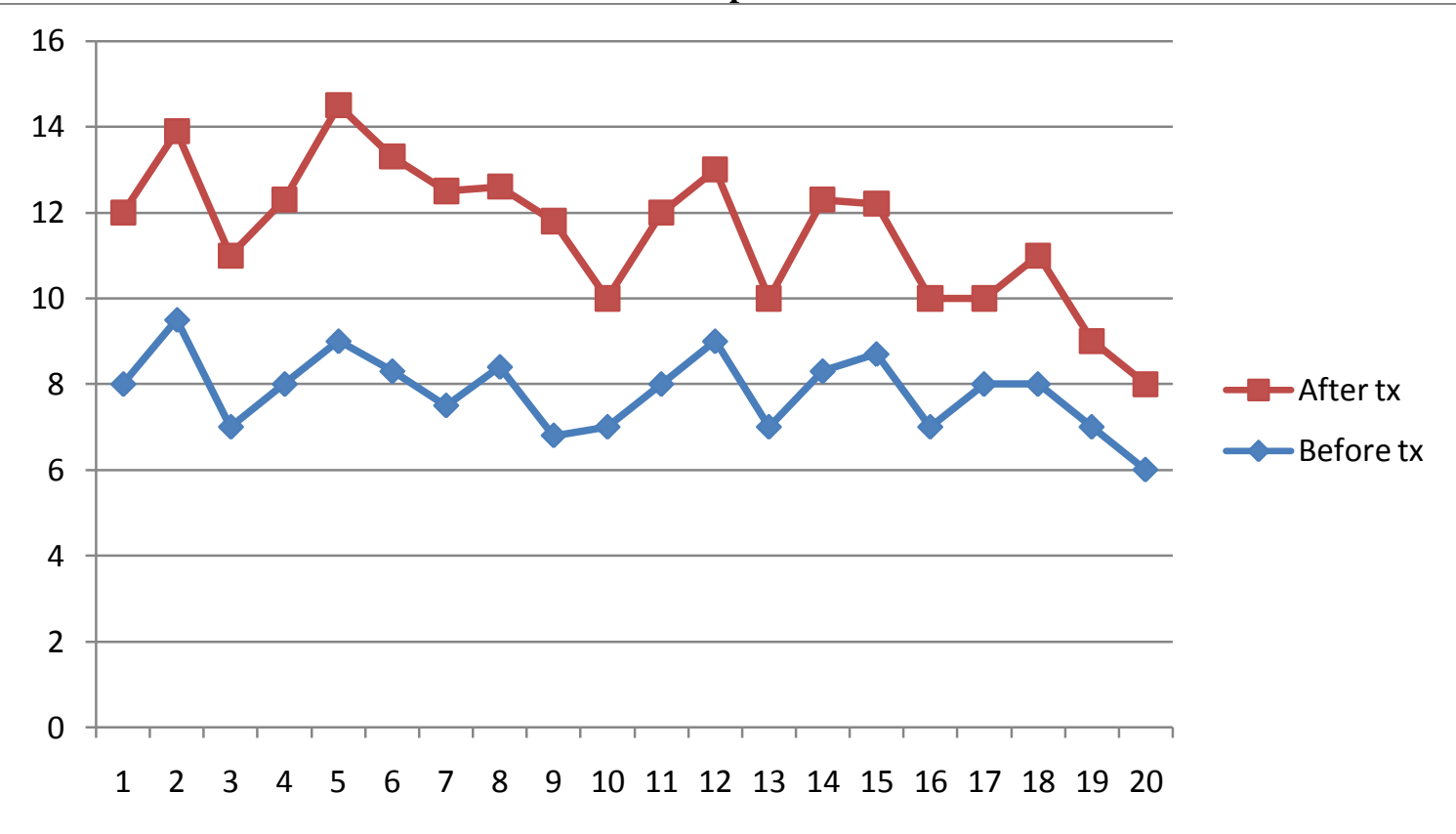

Interpretation: Correlation coefficient $r=0.619$ is more than 0.5 therefore it is positive so there is improvement seen in by using back strengthening exercises. 
Result 3:- McKenzie vs. back strengthening by using VAS

Graph:

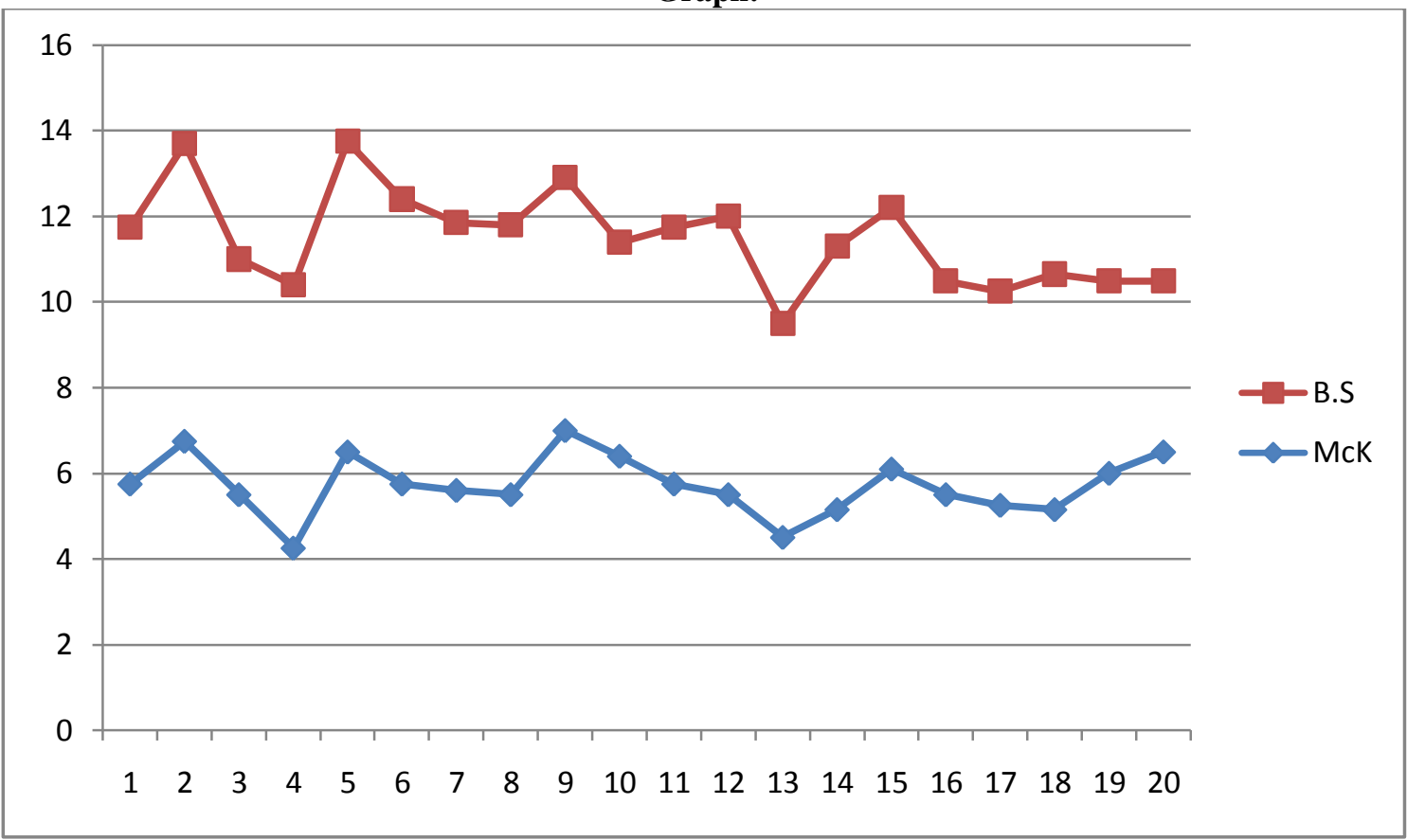

Interpretation: Coefficient of variation of McKenzie treatment method using VAS is 11.9335 is less than coefficient of variation of back strengthening method that is 14.0311 since coefficient of variation of McKenzie is lesser than back strengthening therefore McKenzie is better.

Result 4:- Mean value of Oswestry scale in McKenzie method

Graph:

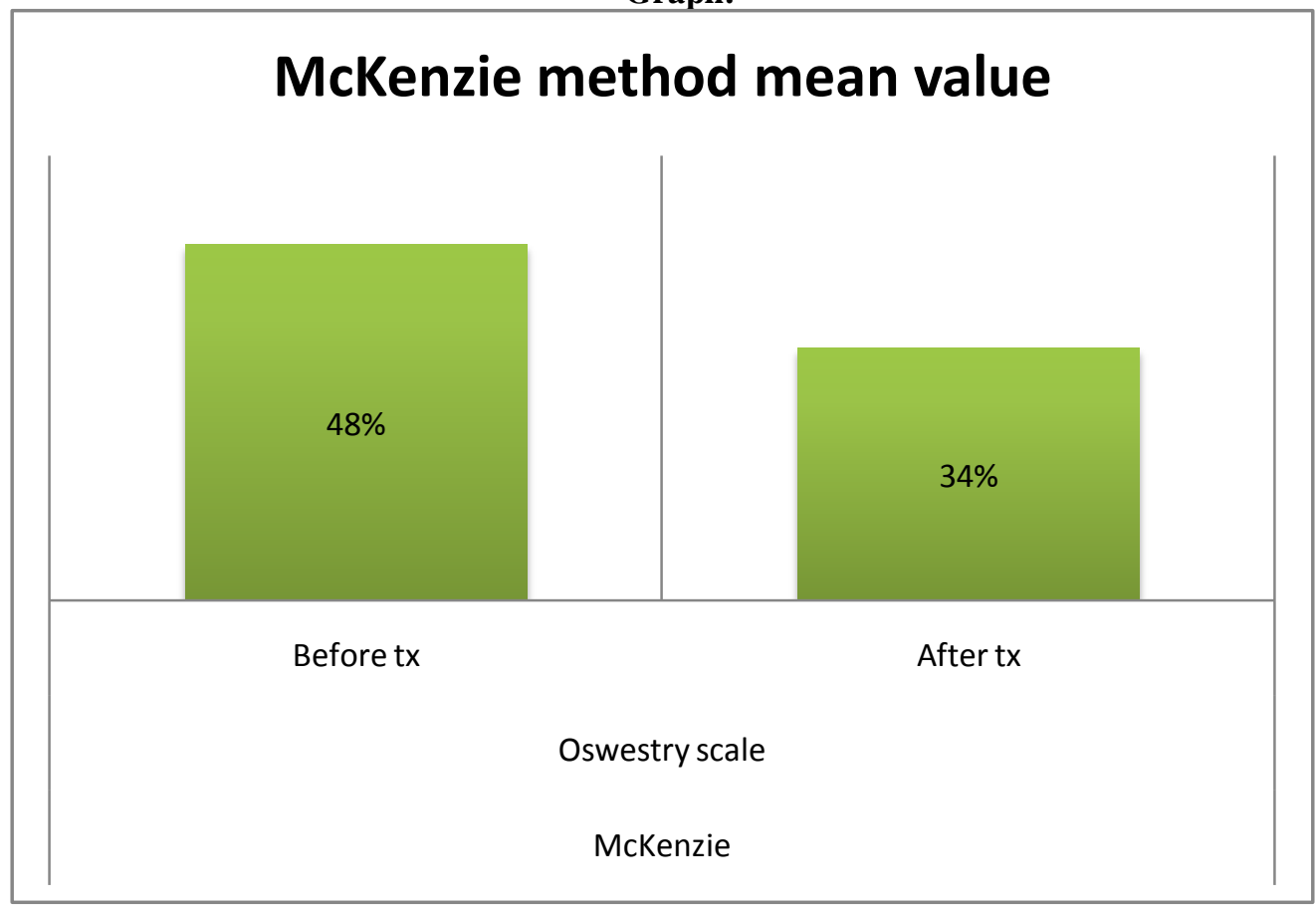

Interpretation: The mean Oswestry scale value of McKenzie method before treatment was $48 \%$ and after the treatment it was 34 
Result 5:- Mean value of Oswestry scale in Back strengthening

Graph:

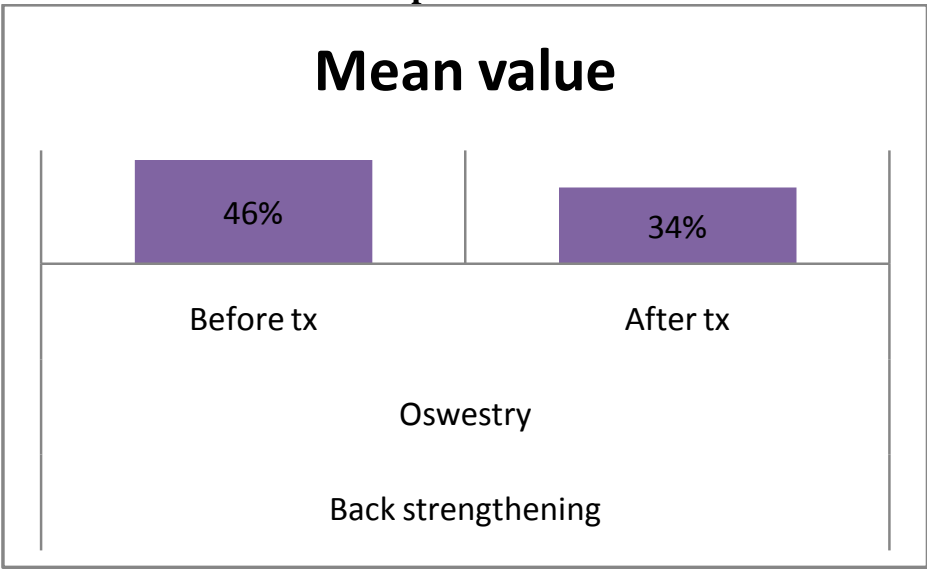

Interpretation: The mean Oswestry scale value of back strengthening method before treatment was $46 \%$ and after the treatment it was $34 \%$.

Result 6:- McKenzie vs. back strengthening by using Oswestry scale

Graph:-

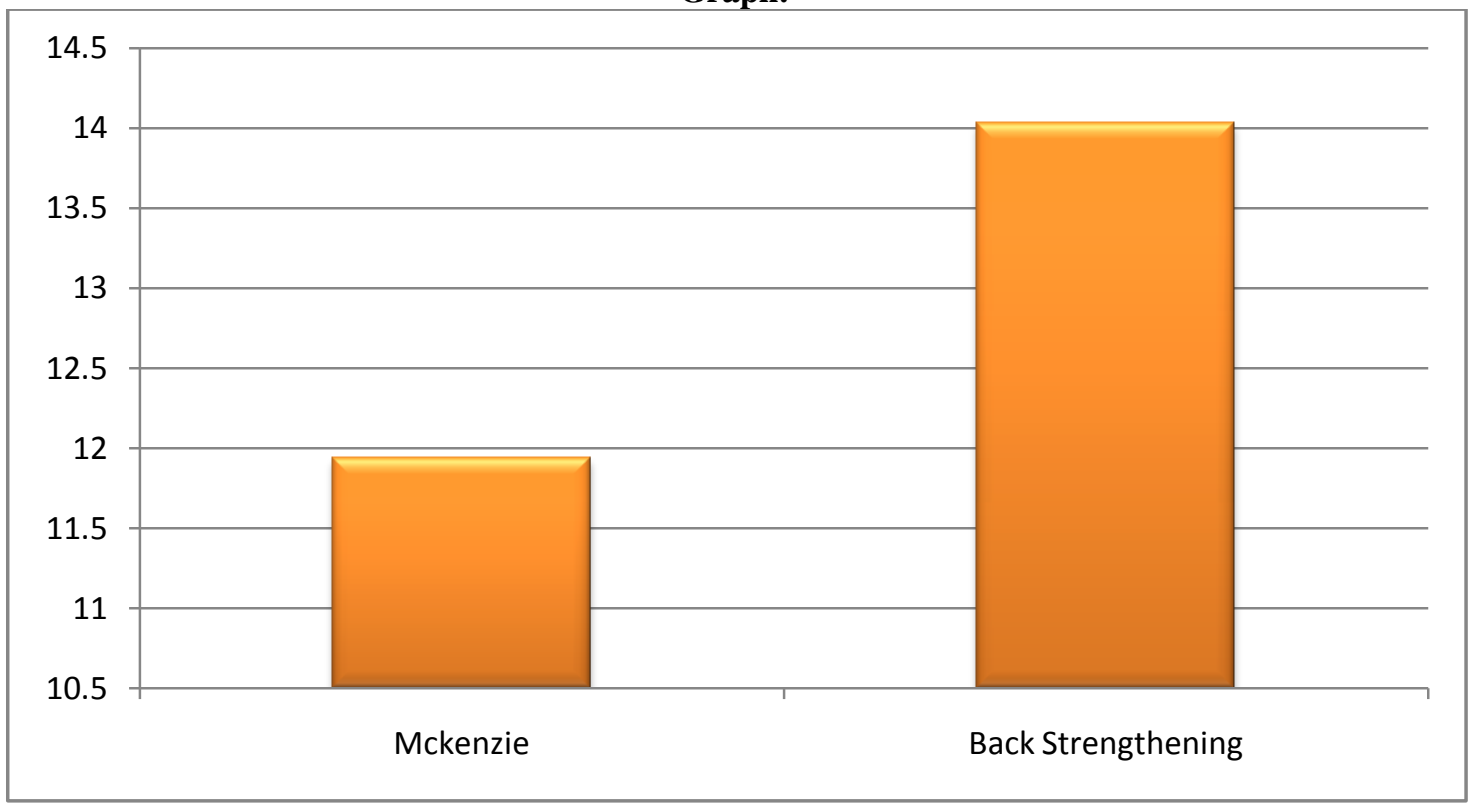

Coefficient of variation of McKenzie treatment method using Oswestry scale is 12.84 is less than coefficient of variation of back strengthening method that is 16.56 .

\section{Discussion:-}

In this study it was found that in McKenzie group the Pain score were considerably lower as compare to back strengthening after completion of the treatment. It seems reasonable to conclude that the McKenzie treatment method has some potential as compared to back strengthening in the treatment of low back pain because the McKenzie treatment method follows a specific pattern of exercises whereas back strengthening exercises are effective but do not follow any pattern.

No previous study has been published comparing McKenzie exercises with strengthening training for low back pain patients. However several recent studies have provided evidence that treatment program containing active exercises are equally effective in patient with chronic low back pain, irrespective of the type of exercises compared. 
Our explanation might maintain that any type of intensive exercise program that manages to make the patients expand the limits to their physical functioning may provide the patients with a method for increasing the feeling of pain control, thus inhibiting negative pain behaviour relating to a chronic low back problem. (3) When done in a controlled, gradual, and progressive manner, active back exercises distribute nutrients into the disc space and soft tissues in the back to keep the discs, muscles, ligaments and joints healthy.

In Tom Petersen et al, the McKenzie method and intensive dynamic strengthening training seems to be equally effective in the treatment of patients with sub-acute or chronic low back pain where as in my study McKenzie method as well as Back strengthening method both were effective but McKenzie treatment method was more effective that back strengthening (3).

In Roger Adams et al, the study says that McKenzie method is effective in back pain which supports this study since in this study McKenzie treatment method was effective and relieved the low back pain (5).

In Keating JL et al, McKenzie exercises were found to be more effective than intensive strengthening for short term and long term pain and short term function, which supports my study(6).

Machado, L. A de Souza et al, suggests that the McKenzie method is more effective than strengthening protocol for acute low back pain which supports the study (7).

Alessandra N Garcia et al suggested that the McKenzie and back school's approaches may be beneficial for the treatment of patients with chronic nonspecific low back pain for the outcomes pain intensity and disability which supports my study. (1) (8)

Based on the results from the articles by Miller et al, spinal stabilization/back strengthening exercises and McKenzie exercises appear equally effective in decreasing pain and disability for individuals with choric low back pain. Miller et al. found statistically significant improvements in pain reduction, for both McKenzie $(p=0.05)$ and spinal Stabilization groups $(p=0.002)$, but no statistically significant differences were detected between groups for pain reduction or disability scores $(p>0.05)$. Poor internal validity, particularly lack of blinding and concealment of group allocation, means that results should very cautiously be applied to the clinical population. (9)

Skikic EM et al suggested that McKenzie exercises for low back pain are beneficial treatment for increasing flexibility of spine and improving the pain with better results in pain relief. Although done by minimally trained physiotherapists in McKenzie approach, McKenzie exercises are successful method for decreasing and centralizing the pain and increasing spinal movements in patients with low back pain which supports my study. (10)

Tarang Srivastava et al, suggested that there was definite decrease in the value of VAS in both the groups but there was more significant improvement in experimental group in comparison to the controlled group. It may be quite safely stated that McKenzie technique is an effective, scientific and economical method for reducing dysfunction pain which supports the study. (11)

Ajit Dabholkar et al says that the effect of extension based treatment program in McKenzie's derangement syndrome improves the recruitment of multifidus on the affected side. Effect of extension based treatment program in McKenzie's derangement syndrome improves the endurance of back extensor muscles. Effect of extension based treatment program in McKenzie's derangement syndrome was found to be functionally beneficial. (12)

Given the outcomes of the current study, the equally distributed amount of time spent with each patient, the types of patients investigated, and the apparent difficulties in proving the effectiveness of a particular type of active exercises over that of others, It seems reasonable to conclude that the McKenzie treatment has some potential, as compared with strengthening training, in the treatment of non-specific chronic low back pain. This conclusion is supported by the results from the supplementary analysis of the patients who completed the full intervention. A strong tendency in favor of the McKenzie treatment was found with regard to a change in the back pain at the end of the treatment that reached a statistically significant difference at the 2 month assessment. (3) (13)

Therefore on the basis of the data, it appears that in managing patients with more than 8 weeks of low back pain, McKenzie treatment method is more effective than compared to back strengthening method. 
Larger sample size can be taken to obtain more accurate results. More such studies should be done by comparing different treatment methods for nonspecific low back pain. If the patient continues the exercises it might give a better and permanent recovery.

\section{References:-}

1. Effectiveness of Back School Versus McKenzie Exercises in Patients With Chronic Nonspecific Low Back Pain: A Randomized Controlled Trial :Alessandra Narciso Garcia, Luci'ola da Cunha Menezes Costa Et al.

2. Van Tulder M, Malmivaara A, Esmail R, et al. Exercise therapy for low back pain: A systemic review within the framework of the Cochrane collaboration back review group, spine 2000;25:2784-96

3. Tom Petersen, Peter Kryger et al. The effect of McKenzie therapy as compared with that of intensive strengthening training for the treatment of patients with acute or chronic low back pain. SPINE Volume 27, Number 16, pp 1702-1709, 2002.

4. Foster NE, Thompson KA, Baxter GD, et al. Management of non- specific low back pain by physiotherapists in Britain and Ireland: A descriptive questionnaire of current clinical practice. Spine 1999;24:1332-42

5. Helen A Clare, Roger Adams et al, A systemic review of efficacy of McKenzie therapy for spine pain. Australian Journal of physiotherapy 2004, vol.50(4):209-216

6. Slade SC, Keating JL et al, Treatment of nonspecific low back pain: McKenzie versus traditional treatment. Journal of Manipulative and physiological therapeutics. 2007;30:301-311.

7. Machao, L. A., de Souza et al, The McKenzie method for low back pain: a systemic review of the literature with a meta-analysis approach. Spine 31, E254-E262

8. Effectiveness of Back School Versus McKenzie Exercises in Patients With Chronic Nonspecific Low Back Pain: A Randomized Controlled Trial :Alessandra Narciso Garcia, Luci'ola da Cunha Menezes Costa Et al.

9. Miller ER, Schnek RJ, Karnes JL, Rousselle JG. A comparison of the McKenzie approach to a specific spine stabilization program for chronic low back pai Journal of Manual and Manipulative Therapy 2005; 12(2):10312.

10. Skikic EM, et al. Bosn J Basic Med Sci 2003. The effects of McKenzie exercises for patients with low back pain, our experience.

11. Tarang Srivastava et al, Efficacy of McKenzie exercise over conventional physiotherapy therapy in ;ow back pain [dysfunction syndrome]

12. Ajit Dabholkar et al, The effect of extension based treatment technique in McKenzie's derangement syndrome on recruitment of multifidus and endurance of back extensor muscles.

13. Tom Petersen, Peter Kryger et al. The effect of McKenzie therapy as compared with that of intensive strengthening training for the treatment of patients with acute or chronic low back pain. SPINE Volume 27, Number 16, pp 1702-1709, 2002.

14. Fairbank JC, Pynsent PB. The Oswestry Disability Index. Spine 2000 Nov 15;25(22):2940-52. 